\title{
Análisis del conocimiento de estudiantes para maestro o maestra en la elaboración de vídeos educativos: una experiencia didáctica
}

\author{
Luis J. Rodríguez-Muñiz*, Marlén Alonso-Castaño y Laura Muñiz-Rodríguez \\ Universidad de Oviedo
}

\author{
PALABRAS CLAVE \\ Educación Primaria \\ Conocimiento especializado \\ Formación inicial \\ del profesorado \\ Transposición didáctica \\ Matemáticas \\ Vídeos educativos
}

\author{
KEYWORDS \\ Primary education \\ Specialized knowledge \\ Initial teacher training \\ Didactic transposition \\ Mathematics \\ Educational videos
}

\begin{abstract}
RESUMEN
Los vídeos educativos están demostrando ser un recurso didáctico con un notable potencial para la mejora del aprendizaje. Por ello, la implementación de estrategias que permitan al futuro profesorado elaborar vídeos educativos de calidad debe estar presente en los programas de formación inicial. Para su elaboración, el profesorado debe movilizar su conocimiento especializado, de cuya idoneidad depende, en gran medida, la calidad educativa del vídeo. En este trabajo se presenta una tarea formativa basada en la elaboración por parte de estudiantes para maestro o maestra (EPM) de un vídeo educativo sobre estadística, probabilidad o resolución de problemas dirigido a alumnado de Educación Primaria. A partir de esta experiencia, se proponen dos objetivos: valorar los vídeos educativos diseñados empleando una rúbrica, y analizar el interés de la tarea formativa para los y las EPM mediante un cuestionario online. Los resultados evidencian, por un lado, una alta representatividad de métodos expositivos y explicaciones deductivas, a menudo acompañadas de ejemplos o situaciones contextualizadas. Por otro lado, los y las EPM mostraron un alto interés por la tarea dada su utilidad en cuanto a su aplicabilidad en su futura práctica docente.
\end{abstract}

\section{Analysis of student teachers' knowledge when elaborating educatio- nal videos: a didactic experience}

\begin{abstract}
Educational videos are proving to be a didactic resource with a remarkable potential to improve learning. Therefore, the implementation of strategies that allow future teachers to elaborate quality educational videos should be present in initial training programs. For its elaboration, teachers must mobilize their specialized knowledge, whose suitability highly influences the educational quality of the video. This work presents a formative task based on the elaboration by student teachers of an educational video on statistics, probability or problem solving aimed at primary school students. Based on this experience, two research goals are proposed: to assess the designed educational videos using a rubric, and to analyze the interest of the formative task for the professional future of the student teachers through an online questionnaire. The results showed, on the one hand, a high representation of expository methods and deductive explanations, often accompanied by examples or contextualized situations. On the other hand, the student teachers showed a high interest in the task given its usefulness in terms of its applicability in their future teaching practice.
\end{abstract}

Universidad de Oviedo

Autor de correspondencia: * Luis J. Rodríguez-Muñiz; luisj@uniovi.es

Recibido: 06/05/2021 - Aceptado: 18/06/2021

Revista de Formación del Profesorado e Investigación Educativa

Facultad de Formación del Profesorado y Educación

Universidad de Oviedo

Enero - Diciembre 2021

ISSN: $2340-4728$

(c) (1) () $\odot$ Esta obra está bajo una licencia internacional Creative Commons

Atribución-NoComercial-SinDerivadas 4.0. 


\section{Introducción}

La identidad profesional de un docente se ve condicionada, entre otros factores, por su formación inicial (Bolívar, 2007; Muñiz-Rodríguez et al., 2020). Uno de los principales objetivos de la formación inicial docente es que el profesorado en formación adquiera las competencias necesarias para desarrollar su futura labor (Darling-Hammond, 2006). El concepto de competencia incluye, además de una serie de habilidades y actitudes, el conocimiento necesario para el desarrollo efectivo de la práctica docente en un contexto multidimensional que abarca al propio docente, sus compañeros, el alumnado, las familias, el centro educativo, y el sistema educativo en su sentido más amplio (Comisión Europea, 2013). La conceptualización del conocimiento del profesorado ha sido objetivo de muchos estudios previos (véase Muñiz-Rodríguez et al., 2020) cuya investigación ha dado como resultado la definición de diferentes modelos teóricos que no han sido ajenos a la transformación de las demandas sociales, las reformas educativas y, en particular, la inmersión de la tecnología en el contexto educativo.

Todos estos modelos se sustentan en la idea precursora de Shulman $(1986,1987)$ basada en la interrelación entre el conocimiento pedagógico y el conocimiento del contenido. Tomando esta teoría como referente, algunos autores y autoras han desarrollado modelos que integran la tecnología como tercera fuente de conocimiento, como el Technological Pedagogical Content Knowledge o modelo TPACK desarrollado por Mishra y Koehler (2006) (véase también Koehler y Mishra, 2009), mientras que otros consideran la necesidad de particularizar el modelo atendiendo a la disciplina a enseñar. Así, para la enseñanza de las matemáticas, nace el $M a-$ thematical Knowledge for Teaching o modelo MKT introducido por Ball et al. (2008) (véase también Hill et al., 2008), el modelo The Knowledge Quartet de Rowland et al. (2005), el Modelo del Conocimiento Didáctico Matemático desarrollado por Godino (2009), o el Mathematics Teachers' Specialized Knowledge o modelo MTSK de Carrillo-Yáñez et al. (2018). Pese a las diferencias derivadas de las particularidades de cada enfoque, todos estos modelos definen el conocimiento especializado del profesorado a partir de una serie de dominios y subdominios que integran características que ya habían sido consideradas por otros autores y autoras con anterioridad y las extienden, como la referente a la noción de transposición didáctica.

La transposición didáctica es definida por Chevallard (1985) como la transformación que experimenta el conocimiento matemático para convertirse en conocimiento escolar. Este proceso es labor del docente y, por tanto, debe formar parte de su conocimiento. En consecuencia, su desarrollo debe estar presente en los programas de formación inicial, a cuya adquisición deben contribuir las personas que forman al profesorado mediante el diseño, implementación y evaluación de tareas formativas. Para que estas tareas formativas sean útiles en cuanto a su aplicabilidad en la futura práctica docente es preciso que incorporen técnicas y herramientas de vanguardia (Gil et al., 2017; Madrid Vivar et al., 2013).

Respecto a la utilidad de las tareas formativas, se debe hacer hincapié en el protagonismo que están cobrando los vídeos educativos en el ámbito escolar y, en particular, en la educación matemática (Beltrán-Pellicer et al., 2018; Myllykoski, 2016). Con frecuencia, estos vídeos educativos constituyen el recurso didáctico que vertebra una orientación metodológica más amplia, como blended learning, flipped learning, mobile learning $\mathrm{u}$ online learning. Estas metodologías, de carácter innovador y consideradas promotoras del aprendizaje activo, comparten el objetivo de aumentar el grado de compromiso del alumnado con su propio aprendizaje, dejando de ser sujeto pasivo que recibe información, para ser sujeto activo que pone en funcionamiento lo que aprende, siendo el protagonista (Smith et al., 2005). Todas ellas se sustentan en el uso de herramientas digitales, que son consideradas dinamizadoras del proceso de enseñanza-aprendizaje, en particular, de las matemáticas, fomentando un aprendizaje autónomo y reflexivo en la adquisición de habilidades matemáticas que, a su vez, fortalecen el autoconcepto de los y las estudiantes (Efklides y Tsiora, 2002; Herrera et al., 2018; Kirmizi, 2015; Ommundsen et al., 2005; Rueda-Gómez, 2020).

Estudios recientes a partir de revisiones bibliográficas y de amplios casos de aplicación (Awidi y Paynter, 2019; Thai et al., 2017) señalan que las metodologías más efectivas son aquellas en las que el alumnado recibe la instrucción por medio de un vídeo educativo acompañado de preguntas que le hagan reflexionar sobre su propio aprendizaje. Además, si consideramos la formación inicial docente, los vídeos educativos son un recurso con un gran potencial, ya que permiten desarrollar competencias docentes en el diseño y desarrollo de entornos educativos y recursos didácticos (Fernández-Río, 2018), así como promover el aprendizaje de la materia (Llinares et al., 2008; Muñiz-Rodríguez et al., 2018) y, en el caso concreto de la matemática, desarrollar el conocimiento matemático especializado.

Para sacar provecho del potencial que los vídeos educativos pueden aportar a la consecución de los objetivos de aprendizaje es preciso que sean de calidad, aspecto no siempre presente en la oferta de vídeos educativos online en canales como YouTube ${ }^{\circledR}$ (Beltrán-Pellicer y Giacomone, 2021; Burgos et al., 2020). Así, se considera indispensable que el profesorado desarrolle una doble capacidad: por un lado, la de seleccionar vídeos educativos adecuados según el contexto y, por otro, la de elaborar vídeos educativos de calidad. Para medir esta calidad es preciso disponer de herramientas que permitan valorar los vídeos educativos en cuanto a la consecución de los objetivos de aprendizaje.

\section{Objetivos}

Se propone una tarea formativa que consiste en elaborar un vídeo educativo sobre estadística, probabilidad o resolución de problemas dirigido a alumnado de Educación Primaria. Un primer objetivo de este trabajo es valorar los vídeos educativos diseñados por los y las EPM para comprobar su conocimiento especializado. Un segundo objetivo consiste en analizar el interés que los y las EPM perciben en la tarea formativa respecto a su futuro profesional.

\section{Metodología}

La tarea formativa se desarrolló en la asignatura "Matemáticas y su Didáctica III" del Grado en Maestro/a en Educación Primaria de la Universidad de Oviedo durante el curso académico 2019-2020. Se trata de una asignatura obligatoria anual de tercer curso de 6 créditos ECTS (European Credit Transfer System). Esta asignatura tiene por objetivo proporcionar a los y las EPM las competencias necesarias para ejercer la docencia de los contenidos curriculares relativos a estadística, probabilidad y resolución de problemas en la etapa de Educación Primaria (6-12 años). Se trata de una asignatura que trata tanto los contenidos matemáticos involucrados como los aspectos didácticos relacionados con ellos (Nolla et al., 2021).

Como tarea formativa en la asignatura se propuso al alumnado la elaboración, por grupos, de un vídeo educativo enmarcado en el bloque de estadística y probabilidad o de resolución de problemas. La tarea había sido planteada en cursos anteriores, por lo que los resultados obtenidos sirvieron para perfilar y definir con mayor concreción los objetivos de aprendizaje, y para enmarcar la creación de los vídeos en el curso 2019-2020 en un Proyecto de Innovación Docente, concedido por la Universidad de Oviedo. A partir de este aprendizaje previo, se estableció que el vídeo debía estar concebido para ser utilizado como un recurso de aprendizaje en formato de aula invertida o flipped learning, es decir, un vídeo que el hipotético alumnado de los y las EPM visualizase antes de una sesión presencial, en el que se tratasen temas no estudia- 
dos anteriormente, y que sirviese como primera aproximación al estudio de un contenido. De este modo, en la sesión presencial se resolverían las dudas que hubiesen podido surgir, pudiendo plantear la resolución de problemas relacionados. El vídeo, que debía tener una duración de entre 5 y 8 minutos, debía indicar en unos títulos de crédito el curso al que iba dirigido y los estándares de aprendizaje evaluables relacionados, así como el software y recursos multimedia utilizados. Como opción de mejora, el vídeo podía estar enriquecido con preguntas o cuestiones planteadas para que el alumnado las resolviese interactivamente durante su visualización.

\section{Recogida y análisis de datos}

Para valorar los vídeos educativos diseñados (primer objetivo de investigación) se diseñó una rúbrica (Tabla 1) compuesta por once indicadores de evaluación organizados en cuatro componentes, con un peso desigual sobre la valoración global del vídeo. Tomando como referencia el modelo de análisis expuesto por Burgos et al. (2020), para valorar cada indicador se utilizó una escala cualitativa compuesta por tres niveles: baja, media o alta (véase en Anexo).

Tabla 1. Componentes e indicadores de la rúbrica para la valoración de los vídeos

\begin{tabular}{|c|c|}
\hline Componente & Indicador \\
\hline \multirow{3}{*}{$\begin{array}{l}\text { Calidad del vídeo } \\
(10 \%)\end{array}$} & Imagen $(2 \%)$ \\
\hline & Sonido $(2 \%)$ \\
\hline & Edición (6 \%) \\
\hline \multirow{3}{*}{$\begin{array}{l}\text { Calidad del contenido } \\
\text { matemático } \\
(40 \%)\end{array}$} & $\begin{array}{l}\text { Adecuación del contenido } \\
\text { matemático }(16 \%)\end{array}$ \\
\hline & $\begin{array}{l}\text { Correspondencia de los estándares } \\
\text { de aprendizaje }(16 \%)\end{array}$ \\
\hline & Claridad y precisión matemáticas (8 \%) \\
\hline \multirow{3}{*}{$\begin{array}{l}\text { Calidad de la transposición } \\
\text { didáctica } \\
(40 \%)\end{array}$} & Calidad del lenguaje matemático ( $8 \%$ ) \\
\hline & $\begin{array}{l}\text { Explicación adecuada al nivel } \\
\text { indicado }(16 \%)\end{array}$ \\
\hline & Recursos utilizados (16 \%) \\
\hline \multirow{2}{*}{$\begin{array}{l}\text { Ampliaciones } \\
(10 \%)\end{array}$} & Nivel de interacción (4%) \\
\hline & Calidad de la interacción (6 \%) \\
\hline
\end{tabular}

Para analizar el interés de la tarea formativa para el futuro profesional de los y las EPM (segundo objetivo de investigación) se diseñó un cuestionario online para ser cumplimentado por el alumnado, administrado a través del campus virtual de la asignatura. El cuestionario incluía un total de cinco preguntas, orientadas a conocer la percepción de dificultad por los y las EPM sobre los distintos procesos que componen la tarea (planificación, guion, búsqueda de recursos, definición de contenidos, grabación, montaje, y coordinación), la suficiencia de las directrices para su realización, las fuentes de información utilizadas y, finalmente, cuatro ítems en escala Likert de 0 a 10 destinados a conocer la valoración de los y las EPM sobre el trabajo en grupo, el interés de la actividad, el interés para su futuro como docentes, y la satisfacción global respecto a la tarea formativa. Para analizar la percepción de dificultades sobre los distintos procesos que componen la tarea se utilizó una escala Likert de 1 a 5 puntos, siendo 1 la mínima y 5 la máxima. En cuanto a las fuentes de información utilizadas, se utilizó una pregunta de opción múltiple de varias respuestas mediante la cual los participantes podían elegir más de una de las fuentes de información proporcionadas (Internet, revistas científicas, libros de texto escolares, libros de texto universitarios, apuntes y material de clase, compañeros, ninguno, $\mathrm{u}$ otros). Además, se incluyó una pregunta abierta mediante la cual los y las EPM podían realizar una valoración personal más extensa. Por tanto, las preguntas incluidas en el cuestionario tienen en cuenta algunas de las sugerencias realizadas por Hidalgo-Moncada et al. (2020) que permiten fomentar el aprendizaje autorregulado de las matemáticas, en nuestro caso, por parte de los y las EPM. A posteriori se realizó un análisis descriptivo de los datos mediante RStudio (versión 1.4.1106).

\section{Resultados}

La tarea formativa fue realizada por 179 de los 196 EPM matriculados en la asignatura, que elaboraron, por grupos, un total de 42 vídeos educativos. Se logró que prácticamente la totalidad del alumnado matriculado en la asignatura $(91.3 \%)$ participase en la experiencia de elaborar su propio vídeo educativo. De los 179 EPM que realizaron el vídeo, 136 respondieron al cuestionario online, lo que supone una tasa de respuesta del $75.9 \%$. Es interesante señalar que la tarea no se planteó como obligatoria dentro de la asignatura, puesto que la valoración de este vídeo supone un $15 \%$ de la calificación final, pero no se exige una nota mínima en este ítem para aprobar la asignatura.

La Figura 1 muestra las valoraciones del profesorado responsable de la asignatura respecto a cada uno de los indicadores de la rúbrica para los vídeos educativos de los y las EPM. Es preciso hacer notar que se trata de un grupo de cuatro profesores y profesoras, con experiencia en la asignatura, y que las posibles discrepancias en la utilización de la rúbrica se fueron resolviendo durante su proceso de aplicación, con el fin de lograr la mayor consistencia posible.

Se observa en la Figura 1 que la calidad de la imagen, del sonido y de la edición del vídeo es media o alta en la mayoría de los casos. De manera puntual, aparecen momentos en algunos vídeos en los que se aprecian cortes en las grabaciones de voz que dejan algunas explicaciones inconclusas, pero debemos tener en cuenta que estas grabaciones se realizaron en los meses de abril-junio de 2020, es decir, en pleno confinamiento por la pandemia COVID-19, lo que limitó los medios técnicos a aquellos de los que los y las EPM disponían en sus domicilios.

En algunos vídeos se aprecia una sobrecarga de texto en algunos fotogramas, que fuerza al espectador a pausar el vídeo para su lectura y comprensión. Siendo la oportunidad de detener la reproducción una de las principales ventajas de los vídeos educativos, es aconsejable cuidar el ajuste entre el contenido escrito y su temporización, de modo que no se conviertan los vídeos en una versión animada de las presentaciones y, heredando, en consecuencia, los problemas educativos que generan (véanse, por ejemplo, De Faoite, 2014; Gurrie y Fair, 2010; Jordan y Papp, 2014).

En cuanto a la calidad del contenido matemático, se determina (Figura 1) que las definiciones y procedimientos están adaptados al nivel educativo al que se dirigen, y el contenido del vídeo está relacionado con los estándares de aprendizaje evaluables correspondientes a los bloques curriculares de resolución de problemas y estadística y probabilidad. Sin embargo, aproximadamente la mitad de los vídeos educativos elaborados presentan imprecisiones o ambigüedades matemáticas que, en algunas ocasiones, dificultan la comprensión o podrían generar aprendizajes erróneos. Por ejemplo, en uno de los vídeos educativos diseñados se explica que "la moda es la variable que más se repite", cuando en realidad la moda es el valor de la variable que más veces se repite. En otro se indica que la variable "número de actividades extraescolares" es cualitativa, cuando realmente su naturaleza es cuantitativa. 


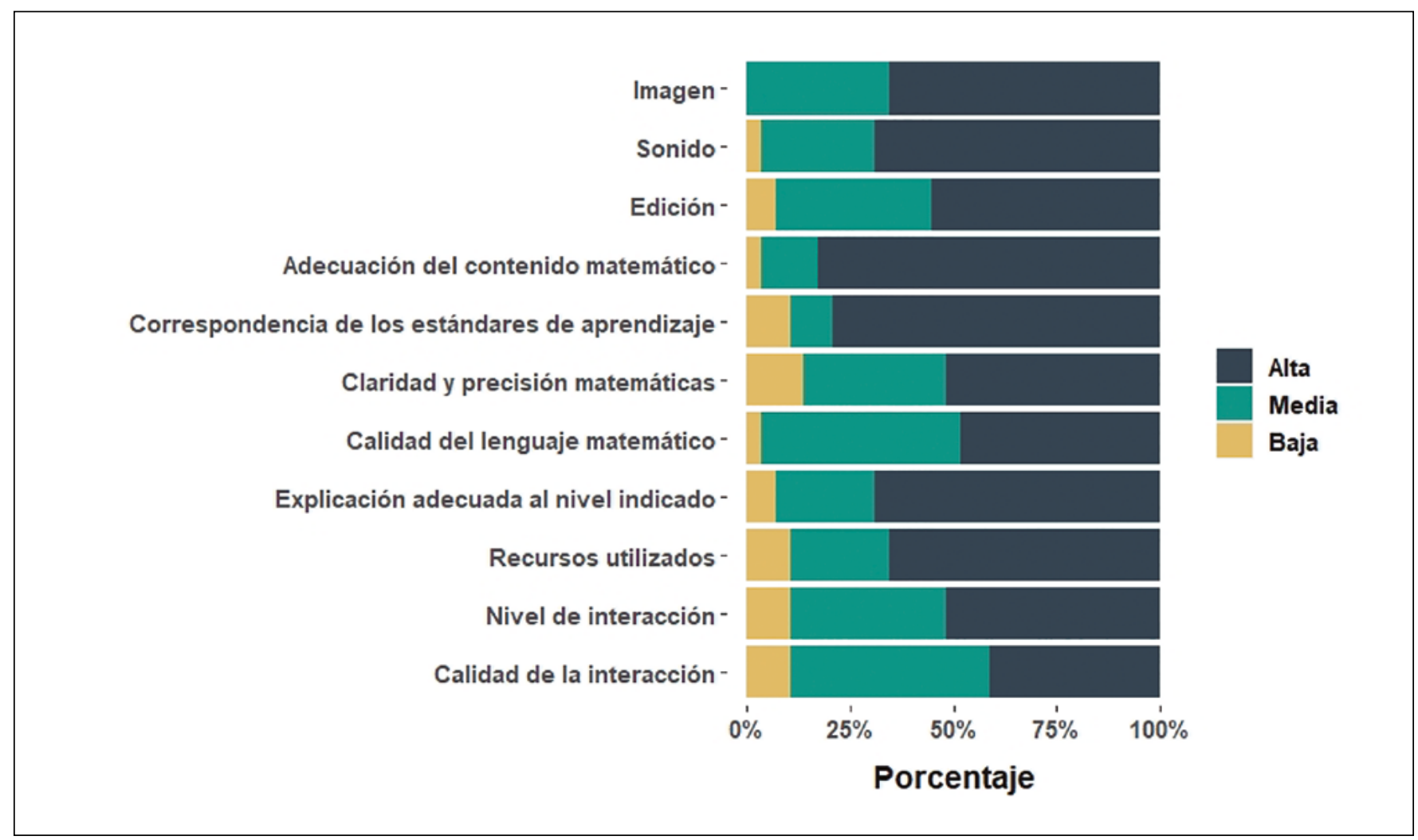

Fuente: Elaboración propia

Figura 1. Valoraciones de los vídeos educativos diseñados

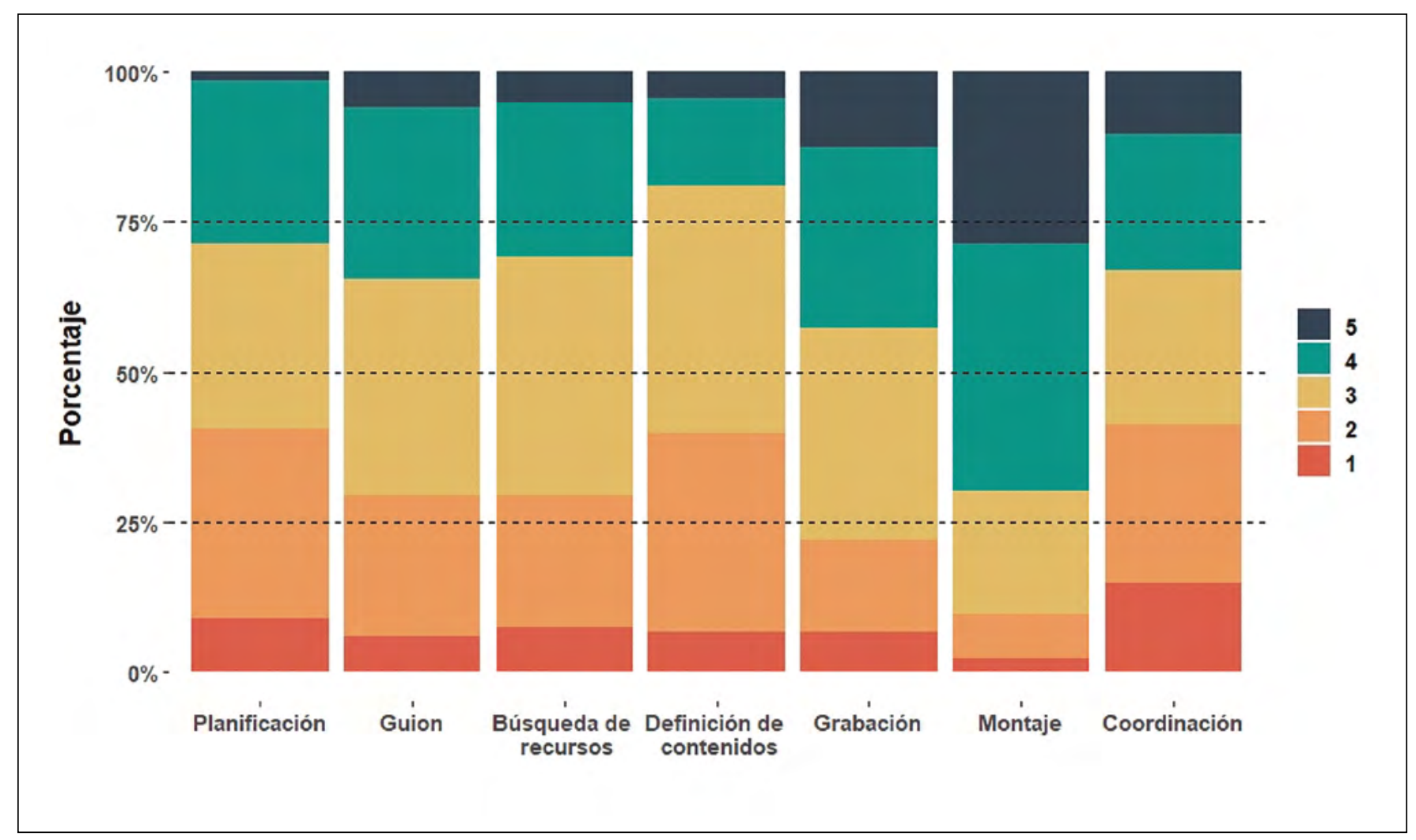

Fuente: Elaboración propia

Figura 2. Valoraciones de los y las EPM sobre la dificultad de la tarea 


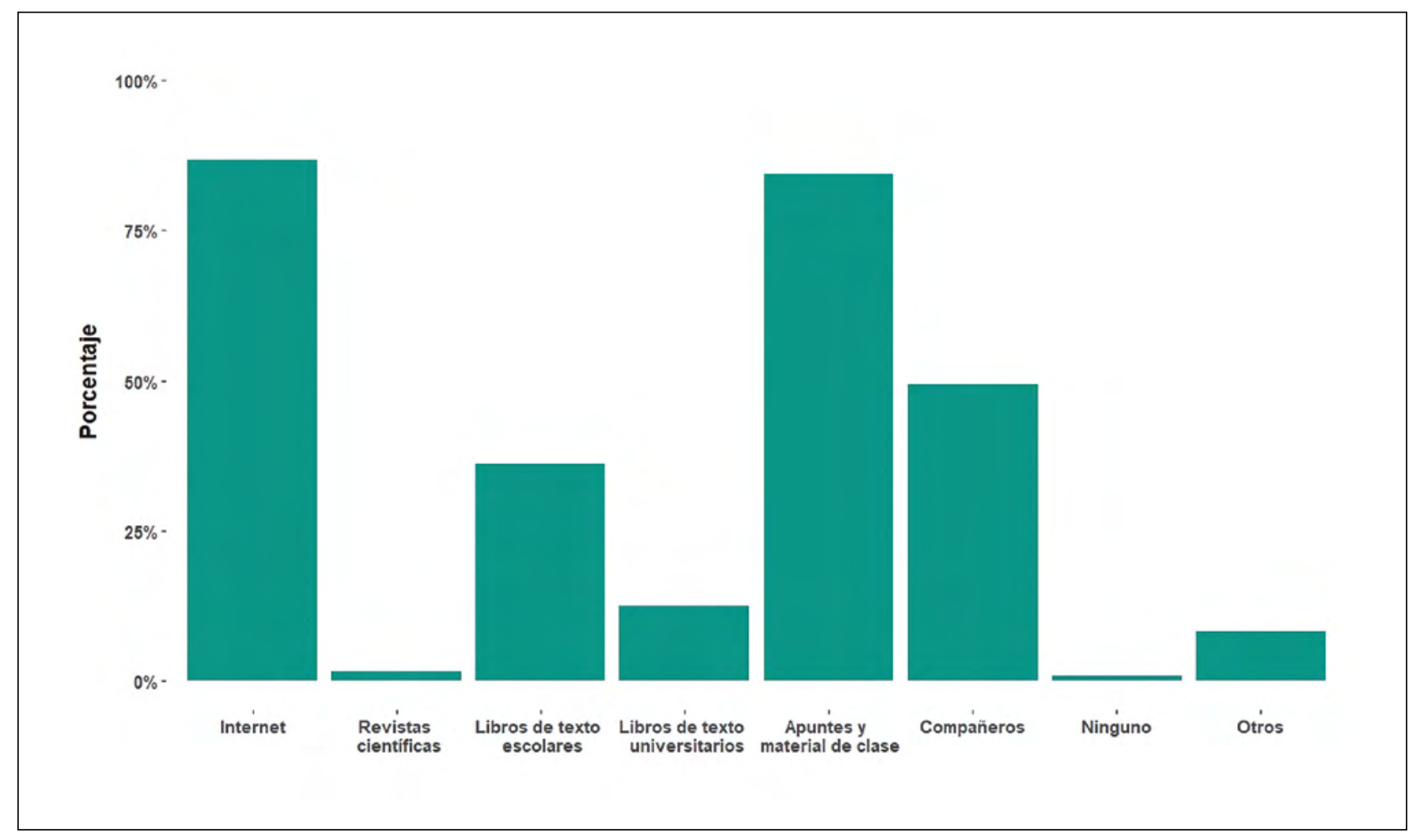

Fuente: Elaboración propia

Figura 3. Fuentes de información utilizadas para elaborar el vídeo

En cuanto a la calidad de la transposición didáctica se observa (Figura 1) que el lenguaje matemático (verbal y escrito) que se utiliza es claro en un $52.4 \%$ de los vídeos. De manera puntual, se maneja algún concepto de manera no rigurosa, vulgarizado, con un exceso de simplificación o complejización, o con una falta de adecuación al nivel al que va dirigido. Por ejemplo, en un vídeo se comenta que "la probabilidad se define como la posibilidad de que ocurra algo", lo cual es claramente una definición inadecuada por ser demasiado circular. Por otro lado, la argumentación, el razonamiento y las explicaciones que se proporcionan en casi la tercera parte de los vídeos parten del conocimiento previo del alumnado ayudándole a adquirir nuevos conceptos, utilizando contextos y situaciones cercanas y de interés, sin caer en una excesiva simplificación. Además, se percibe una alta representatividad de métodos expositivos y explicaciones deductivas, casi siempre acompañadas de ejemplos o situaciones contextualizadas. En relación con los recursos utilizados, se observa una alta representación de recursos manipulativos físicos o digitales para ayudar a construir los conceptos matemáticos que se trabajan, para representar el problema que se propone o para buscar su solución siendo, en la mayoría de los casos, adecuados para la etapa correspondiente.

Por último, en cuanto al nivel y calidad de las interacciones, el $83.3 \%$ de los vídeos educativos elaborados plantean actividades y preguntas (Figura 1), pero solo en la mitad de ellos se establece algún medio para comprobar las respuestas (en el resto, el vídeo se limita a plantear preguntas o actividades), principalmente a través de software de enriquecimiento de vídeos (como edpuzzle ${ }^{\circledR}$ o Genially $\left.{ }^{\circledR}\right)$. Además, en algunos vídeos se incluyen preguntas relacionadas con conceptos que no han sido mencionados ni explicados en el vídeo educativo, lo cual puede confundir al alumnado. En un $40.5 \%$ de los vídeos, las preguntas se plantean verbalmente mientras que, en otro $40.5 \%$, se realiza utilizando software específico de edición de vídeos.
Como se observa en la Figura 2, la dificultad de cada uno de los procesos que componen la tarea fue variada en cinco de los aspectos y un poco más diferente en lo que respecta a la grabación y el montaje. Se perciben valoraciones más bajas en lo que se refiere a la planificación, la definición de contenidos y la coordinación, y más altas en relación con la grabación y el montaje. Asimismo, un $90.4 \%$ de los participantes indicaron que las directrices proporcionadas para realizar la tarea fueron suficientes.

En cuanto a las fuentes de información utilizadas para la elaboración del vídeo educativo (Figura 3), todos los y las EPM menos uno, reconocieron consultar alguna fuente de información para realizar la tarea formativa. Prevaleció el uso de Internet y de los apuntes y material de clase, empleados por el $86.8 \%$ y 84.6 $\%$ de los participantes, respectivamente, seguidos de la consulta a sus compañeros de clase ( $49.3 \%$ ) y de libros de texto escolares (36\%). Por el contrario, se observa un escaso empleo de libros de texto universitarios $(12.5 \%)$, de otros materiales $(8.1 \%)$, y de revistas científicas $(1.5 \%)$.

La Tabla 2 muestra las valoraciones medias y medianas de los participantes sobre la tarea formativa. La satisfacción global con la tarea formativa alcanzó una calificación media casi sobresaliente.

Tabla 2. Valoraciones medias de los y las EPM sobre la tarea

\begin{tabular}{|l|c|c|}
\hline Indicador & Media & Mediana \\
\hline Trabajo en grupo & 8.8 & 9 \\
\hline Interés de la actividad & 8.2 & 8 \\
\hline Interés para su futuro como docente & 8.4 & 9 \\
\hline Satisfacción global de la tarea formativa & 8.7 & 9 \\
\hline
\end{tabular}


Todas las respuestas abiertas, salvo una, son positivas respecto a la utilidad de la experiencia. Con sus comentarios, los y las EPM demostraron una gran madurez metacognitiva, al ser capaces de reflexionar sobre su propio proceso de aprendizaje y reconocer que el hecho de tener que enfrentarse a la elaboración de un vídeo educativo les ayudó a plantearse muchas cuestiones sobre lo que creían que sabían y lo que realmente sabían, tanto de matemáticas como de didáctica de la matemática, tal y como se aprecia en las siguientes valoraciones individuales: "al principio no pensé que este trabajo me fuese a proporcionar mucho aprendizaje, nunca habíamos trabajado flipped classroom y no sabíamos muy bien cómo enfocarlo. Sin embargo, una vez fuimos dándole forma al trabajo, me di cuenta de que era un recurso muy interesante para Primaria [énfasis añadido] y que tenía muchos puntos fuertes", "me parece una actividad divertida y entretenida, que nos ayuda a trabajar contenidos, definiciones y aprender a desarrollar problemas de cara al futuro en un colegio. Sí que es cierto que en momentos se presentaron una serie de dificultades [énfasis añadido], pero creo que supimos resolverlas de manera eficiente" o "resulta de vital importancia [énfasis añadido] que se desarrollen este tipo de trabajos, pues no dejan de ser ensayos de elaboración de recursos que podemos poner en práctica nuestro futuro".

\section{Discusión y conclusiones}

Este estudio muestra el conocimiento matemático especializado de los y las EPM a la hora de elaborar un vídeo educativo sobre estadística, probabilidad o resolución de problemas dirigido al alumnado de Educación Primaria. De las valoraciones realizadas por el profesorado que imparte la asignatura, se evidencia una calidad alta en al menos la mitad de los vídeos educativos diseñados tanto en lo relativo a los aspectos técnicos del vídeo como al contenido matemático y a la transposición didáctica realizada por los y las EPM. Sin embargo, existe un grupo importante de EPM que muestra evidencias de un conocimiento matemático y una transposición didáctica débil en sus vídeos educativos. Este resultado respalda la necesidad de incidir en la formación matemática de los y las EPM en los programas de formación inicial para mejorar su conocimiento especializado, que incluye tanto el conocimiento del contenido matemático como el conocimiento de la didáctica de la matemática (Carrillo-Yáñez et al., 2018), en particular, en lo referente a su razonamiento estadístico y probabilístico, y a la resolución de problemas (Arteaga et al., 2017; López-Beltrán et al., 2020).

Resulta de interés comentar algunas de las dificultades encontradas por los y las EPM al realizar la tarea y su relación con las fuentes de información consultadas. Por un lado, Melguizo-Moreno y Fernández-Plaza (2019) señalan que la selección de contenidos matemáticos como parte del proceso de transposición didáctica es un aspecto complejo que en ocasiones genera discrepancias entre los investigadores y los futuros docentes. En contraposición, la concreción de los contenidos matemáticos fue uno de los procesos de la tarea valorados por los y las EPM como de menor dificultad en este estudio. Esta divergencia entre resultados hace que el tratamiento de este proceso resulte de mayor interés en el periodo de formación inicial docente, no tanto en cuanto a qué contenidos son o no seleccionados, sino a las razones que puedan argumentar los y las EPM para su inclusión o rechazo, y a los factores que implícitamente estén confrontando su competencia y su percepción.

A su vez, en la concreción de contenidos, el tipo de fuentes de información consultadas juega un papel relevante. Los resultados de este estudio evidencian una escasa utilización de revistas científicas. Ahora bien, en las publicaciones en formato de artículo aparecen reflexiones relevantes tanto de investigadores como de docentes, que pueden documentar la toma informada de decisiones con respecto al contenido. Por tanto, parece necesario promover en la formación inicial docente la consulta de estas fuentes y concienciar a los y las EPM sobre su relevancia. En este sentido, existe en la literatura un claro consenso al reconocer el conocimiento de recursos para docentes como revistas científicas y de divulgación en didáctica de las matemáticas, páginas web de asociaciones matemáticas, entre otros, como una competencia docente clave (Muñiz-Rodríguez et al., 2017).

Resulta curiosamente sorprendente que, a pesar de haber utilizado durante la asignatura dos manuales universitarios como libros de texto (Batanero y Godino, 2002; Blanco Nieto et al., 2015), apenas un $12.5 \%$ de los y las estudiantes refieran haberlo tenido como material de consulta para la elaboración del vídeo. Sin embargo, no sorprende que uno de los mayores apoyos en lo referente a las fuentes de información, por detrás de las consultas a través de Internet, sean las consultas entre los propios compañeros, dada la naturaleza grupal del trabajo. Asimismo, se considera positivo la gran cantidad de vídeos que utilizan recursos y materiales manipulativos físicos o virtuales para apoyar las explicaciones, puesto que es un aspecto sobre el cual se incide mucho en la asignatura (Rodríguez-Muñiz et al., 2021). No obstante, también en la asignatura se subraya la importancia de la inducción en la construcción del conocimiento matemático, con una aproximación basada en la matemática realista (Freudenthal, 2002; Van den Heuvel-Panhuizen y Drijvers, 2020), que no se ve reflejada en una gran mayoría de vídeos con una aproximación muy deductiva a los conceptos matemáticos. Parece que, a pesar del modo en el que han sido instruidos, los y las EPM parten de una concepción rígida respecto a qué debe ser una clase de matemáticas. Por otro lado, consideramos positivo el alto porcentaje de vídeos que incorporan cuestiones interactivas, pues están relacionadas con la evaluación formativa, otra cuestión a la que se dedica tiempo en la asignatura.

Como conclusión, la tarea formativa implementada favorece el desarrollo de competencias docentes en cuanto al diseño de recursos didácticos y a la adquisición de conocimiento matemático especializado por los y las EPM, respondiendo así a las demandas actuales (Fernández-Río, 2018; Llinares et al., 2008; Muñiz-Rodríguez et al., 2018). Esta apreciación coincide con la percepción de los y las EPM, para quienes la elaboración de vídeos educativos ha resultado ser de utilidad para su futuro docente, hecho que anima a replicar la experiencia en futuros cursos académicos, susceptible a la incorporación de alguna mejora.

La rúbrica utilizada en este estudio ha permitido analizar la calidad del conocimiento matemático especializado y de la transposición didáctica de los y las EPM desde una perspectiva general, pudiendo esto ser considerado como una de las limitaciones del trabajo. A raíz de los resultados obtenidos, parece necesario realizar un análisis más exhaustivo de los vídeos educativos que permita valorar con mayor detalle algunos aspectos epistémicos y cognitivos, con la idea de refinar la tarea formativa en futuros cursos académicos y mejorar el conocimiento especializado de los y las EPM durante su formación inicial. En este sentido, recientes investigaciones han diseñado y validado herramientas que permiten valorar la medida en que un recurso didáctico, como un vídeo educativo, puede ser considerado óptimo en cuanto a la enseñanza del contenido matemático. Así, se parte de la definición de la idoneidad didáctica de Godino (2013) como herramienta para valorar la medida en que un proceso matemático de enseñanza y aprendizaje puede ser considerado como óptimo, a partir de seis facetas: epistémica, cognitiva, interaccional, mediacional, afectiva, y ecológica. Cada una de estas facetas se sustenta, a su vez, en una serie de componentes empíricas que permiten medir la calidad del recurso didáctico. Algunos autores y autoras han concretado estas componentes de manera empírica mediante la definición de indicadores que permiten valorar la idoneidad didáctica de un contenido matemático específico (Beltrán-Pellicer y Giacomone, 2021; Burgos et al., 2020). En particular, Beltrán-Pellicer y Giacomone (2021) concretan estos indicadores para el caso de la probabilidad. De manera similar, pero con un enfoque ligeramente 
distinto, Vásquez et al. (2020) diseñan y validan un instrumento para la observación de clases de probabilidad en Educación Primaria, que también puede ser utilizado para la valoración de vídeos educativos. Siguiendo el perfil de una rúbrica, Vásquez et al. (2020) consideran cinco dimensiones (tareas, razonamiento, conexiones, comunicación, y lenguaje probabilístico), para cada una de las cuales se definen una serie de componentes con sus respectivos niveles de ejecución (bajo, medio bajo, medio alto, alto). Las autoras y autor del presente estudio ya han comenzado a trabajar en esta línea (Muñiz-Rodríguez et al., 2021), seleccionando los vídeos educativos diseñados por los y las EPM sobre probabilidad y haciendo un análisis más exhaustivo del conocimiento matemático especializado movilizado por los y las EPM, utilizando tanto los indicadores definidos por Beltrán-Pellicer y Giacomone (2021) como por Vásquez et al. (2020). Un análisis preliminar revela que la idoneidad de la mayoría de los vídeos educativos es media o baja, a excepción del indicador relativo a la adecuación del nivel lingüístico. De acuerdo con Hidalgo-Moncada et al. (2020) sobre la necesidad de promover la autorregulación de los y las EPM, otra mejora implementada a la tarea formativa en el curso académico 2020-2021 pasa por informar a los y las EPM sobre los indicadores de idoneidad didáctica con los que se va a valorar el vídeo educativo elaborado de modo que permita a los y las EPM reflexionar sobre aquellos aspectos epistémicos y cognitivos más destacados.

\section{Agradecimientos}

Se quiere hacer constar el apoyo de los Proyectos de Innovación PINN-19-A-019 y PINN-20-B-008, de la Universidad de Oviedo y del Proyecto TIN2017-87600-P del Ministerio de Ciencia e Innovación de España. Las autoras y el autor quieren agradecer a la profesora Esther Lorenzo y el profesor Antón Lombardero por su colaboración en la experiencia y a todo el alumnado participante por su interés y sus comentarios.

\section{Contribución individual de las autoras y autor al manuscrito}

LJRM: conceptualización, metodología, recogida y tratamiento de los datos, análisis, correcciones y edición, coordinación. MAC: conceptualización, metodología, recogida y tratamiento de los datos, análisis, correcciones y edición, software. LMR: conceptualización, metodología, análisis, escritura original y visualización.

\section{Referencias}

Arteaga, P., Gea, M.M., y Batanero, C. (2017). La componente mediacional del conocimiento didáctico-matemático de futuros profesores sobre Estadística: un estudio de evaluación exploratorio. Educação Matemática Debate, 1(1), 54-75. http://dx.doi. org/10.24116/emd25266136v1n12017a03

Awidi, I.T., y Paynter, M. (2019). The impact of a flipped classroom approach on student learning experience. Computers $\mathcal{E}$ Education, 128, 269-283. https://doi.org/10.1016/j.compedu.2018.09.013

Ball, D.L., Thames, M.H., y Phelps, G. (2008). Content knowledge for teaching: What makes it special? Journal of Teacher Education, 59(5), 389-407. https://doi.org/10.1177/0022487108324554

Batanero, C., y Godino, J. D. (2002). Estocástica y su didáctica para maestros. Departamento de Didáctica de la Matemática.Universidad de Granada.

Beltrán-Pellicer, P., Giacomone, B., y Burgos, M. (2018). Online educational videos according to specific didactics: the case of mathematics. Cultura y Educación, 30(4), 633-662. https://doi. org/10.1080/11356405.2018.1524651

Beltrán-Pellicer, P., y Giacomone, B. (2021). Una primera aproximación al análisis de vídeos educativos de estadística: el caso de la mediana. Números, 106, 53-61.
Blanco Nieto, L.J., Cárdenas Lizarazo, J.A., y Caballero Carrasco, A. (2015). La resolución de problemas de Matemáticas en la formación inicial de profesores de Primaria. Universidad de Extremadura.

Bolívar, A. (2007). La formación inicial del profesorado de secundaria y su identidad profesional. Estudios sobre Educación, 12, 13-30. https://hdl.handle.net/10171/8987

Burgos, M., Beltrán-Pellicer, P., y Godino, J.D. (2020). La cuestión de la idoneidad de los vídeos educativos de matemáticas: una experiencia de análisis con futuros maestros de educación primaria. Revista Española de Pedagogía, 78(275), 27-49. https://doi. org/10.22550/rep78-1-2020-07

Carrillo-Yáñez, J., Climent, N., Montes, M., Contreras, L.C., Flores-Medrano, E., Escudero-Ávila, D., Vasco, D., Rojas, N., Flores, P., Aguilar-González, Á., Ribeiro, M. y Muñoz-Catalán, M.C. (2018). The mathematics teacher's specialized knowledge (MTSK) model. Research in Mathematics Education, 20(3), 236253. https://doi.org/10.1080/14794802.2018.1479981

Chevallard, Y. (1985). La transposition didactique; du savoir savant au savoir enseigné. La Pensée Sauvage.

Comisión Europea (2013). Supporting teacher competence development for better learning outcomes. Comisión Europea.

Darling-Hammond, L. (2006). Constructing 21st-century teacher education. Journal of Teacher Education, 57(3), 300-314. https:// doi.org/10.1177/0022487105285962

De Faoite, D. (2014). Getting more out of PowerPoint ${ }^{\circledR}$. Medical Writing, 23(1), 22-24. https://doi.org/10.1179/204748061 3Z.000000000180

Efklides, A., y Tsiora, A. (2002). Metacognitive experiences, self-concept, and self-regulation. Psychologia, 45(4), 222-236. https://doi.org/10.2117/psysoc.2002.222

Fernández-Río, J. (2018). Creación de vídeos educativos en la formación docente: un estudio de caso. Revista electrónica interuniversitaria de formación del profesorado, 21(1), 115-127. http:// dx.doi.org/10.6018/reifop.21.1.293121

Freudenthal, H. (2002). Revisiting mathematics education: China lecture. Kluwer.

Gil, M.J., Martínez, M.B., y Cordero, S. (2017). Grabaciones de situaciones de aula para la formación del profesorado. Ápice. Revista de Educación Científica, 1(1), 58-73. https://doi. org/10.17979/arec.2017.1.1.2005

Godino, J.D. (2009). Categorías de análisis de los conocimientos de profesor de matemáticas. UNIÓN, 20, 13-31.

Godino, J.D. (2013). Indicadores de idoneidad didáctica de procesos de enseñanza y aprendizaje de las matemáticas. Cuadernos de Investigación y Formación en Educación Matemática, 11, 111-132.

Gurrie, C., y Fair, B. (2010). PowerPoint--from fabulous to boring: The misuse of PowerPoint in higher education classrooms. Journal of the Communication, Speech, and Theatre Association of North Dakota, 23, 23-30.

Herrera, M. A., Fernández, D. C., y Seguel, R. C. (2018). Percepción de los profesores sobre integración de TIC en las prácticas de enseñanza en relación a los marcos normativos para la profesión docente en Chile. Ensaio: Avaliação e Políticas Públicas em Educação, 26(98) 163-184. http://dx.doi.org/10.1590/ s0104-40362017002501119

Hidalgo-Moncada, D., Díez-Palomar, J., y Vanegas Muñoz, Y. (2020). Formación de maestros de educación primaria en el contexto de confinamiento. La importancia del aprendizaje autorregulado en las matemáticas. Magister 32, 40-48. https:// doi.org/10.17811/msg.32.1.2020.40-48

Hill, H.C., Ball, D.L., y Schilling, S.G. (2008). Unpacking pedagogical content knowledge: Conceptualizing and measuring teachers' topic-specific knowledge of students. Journal for Research in Mathematics Education, 39(4), 372-400. https://doi. org/10.5951/jresematheduc.39.4.0372 
Jordan, L. A., y Papp, R. (2014). Powerpoint ${ }^{\circledR}$ : It's Not" Yes" or" No"--It's" When" and" How". Research in Higher Education Journal, 22. https://files.eric.ed.gov/fulltext/EJ1064139.pdf

Kirmizi, Ö. (2015). The interplay among academic self-concept, self-efficacy, self-regulation and academic achievement of higher education L2 learners. Journal of Higher Education and Science, 5(1), 32-40. https://dergipark.org.tr/en/pub/higheredusci/issue/61486/918077

Koehler, M.J. y Mishra, P. (2009). What is technological pedagogical content knowledge? Contemporary Issues in Technology and Teacher Education, 9(1), 60-70. https://www.learntechlib. org/primary/p/29544/

Llinares, S., Valls, J., y Roig, A.I. (2008). Aprendizaje y diseño de entornos de aprendizaje basado en videos en los programas de formación de profesores de matemáticas. Educación Matemática, 20(3), 59-82.

López Beltrán, M., Albarracín, L., Ferrando-Palomares, I., Montejo-Gámez, J., Ramos, P., Serradó, A., Thibaut, E., y Mallavibarrena, R. (2020). La educación matemática en las enseñanzas obligatorias y el Bachillerato. En D. Martín De Diego, T. Chacón, G. Curbera, F. Marcellán y M. Siles (Eds.), Libro Blanco de las Matemáticas (pp. 1-94). Editorial Centro de Estudios Ramón Areces. https://www.fundacionareces.es/fundacionareces/es/ publicaciones/libro-blanco-de-las-matematicas.html

Madrid Vivar, D., Mayorga Fernández, M., y Núñez Avilés, F. (2013). Aplicación del m-learning en el aula de primaria: Experiencia práctica y propuesta de formación para docentes. Edutec. Revista Electrónica de Tecnología Educativa, 45, 1-12. https://doi.org/10.21556/edutec.2013.45.27

Melguizo-Moreno, E., y Fernández-Plaza, J.A. (2019). La transposición didáctica de contenidos de Lengua Castellana y Literatura y Matemáticas desde la perspectiva de los maestros en formación de Educación Primaria. Revista Internacional de Educación y Aprendizaje, 6, 259-274. https://doi.org/10.37467/ gka-revedu.v6.1855

Mishra, P. y Koehler, M.J. (2006). Technological Pedagogical Content Knowledge: A framework for teacher knowledge. Teachers College Record, 108(6), 1017-1054. https://doi.org/10.1111/j.14679620.2006.00684.x

Muñiz-Rodríguez, L., Alonso, P., Rodríguez-Muñiz, L.J., y Valcke, M. (2017). Developing and validating a competence framework for secondary mathematics student teachers through a Delphi method. Journal of Education for Teaching, 43(4), 383399. https://doi.org/10.1080/02607476.2017.1296539

Muñiz Rodríguez, L., Alonso, P., Rodríguez-Muñiz, L.J., De Coninck, K., Vanderlinde, R., y Valcke, M. (2018). Exploring the effectiveness of video-vignettes to develop mathematics student teachers' feedback competence. Eurasia Journal of Mathematics Science and Technology Education, 14(11), em1573. https:// doi.org/10.29333/ejmste/92022

Muñiz-Rodríguez, L., Aguilar-González, Á. y Rodríguez-Muñiz, L.J. (2020). Perfiles del futuro profesorado de matemáticas a partir de sus competencias profesionales. Enseñanza de las Ciencias, 38(2), 141-161. https://doi.org/10.5565/rev/ensciencias.3161
Muñiz-Rodríguez, L., Alonso-Castaño, M., y Rodríguez-Muñiz, L. J. (2021). Análisis de la idoneidad didáctica de vídeos educativos sobre probabilidad elaborados por estudiantes para maestro. En P. D. Diago, D.F. Yáñez, M.T. González-Astudillo y D. Carrillo (Eds.), Investigación en Educación Matemática XXIV (pp. 449-456). SEIEM https://www.seiem.es/docs/actas/24/ActasXXIVSEIEM.pdf

Myllykoski, T. (2016). Educational videos and use of tools in mathematics remedial instruction (Trabajo Final de Máster). Tampere University of Technology. https://cutt.ly/JxSLJ6X

Nolla, A., Muñoz, R., Cerisola, A., y Fernández, B. (2021). La formación inicial de los maestros en matemáticas y su didáctica. Revista Interuniversitaria de Formación del Profesorado, 96(35.1),185-208. https://doi.org/10.47553/rifop.v96i35.1.85882

Ommundsen, Y., Haugen, R., y Lund, T. (2005). Academic selfconcept, implicit theories of ability, and self-regulation strategies. Scandinavian Journal of Educational Research, 49(5), 461474. https://doi.org/10.1080/00313830500267838

Rodríguez-Muñiz, L.J., Muñiz-Rodríguez, L., y Aguilar, Á. (2021). El recuento y las representaciones manipulativas: los primeros pasos de la alfabetización estadística. PNA, Revista de Investigación en Didáctica de la Matemática. En prensa.

Rowland, T., Huckstep, P., y Thwaites, A. (2005). Elementary teachers' mathematics subject knowledge: the knowledge quartet and the case of Naomi. Journal of Mathematics Teacher Education, 8(3), 255-281. https://doi.org/10.1007/s10857005-0853-5

Rueda-Gómez, K.L. (2020). Estrategia educativa remota en tiempos de pandemia. Magister 32, 93-96. https://doi.org/10.17811/ msg.32.1.2020.93-96

Shulman, L. (1986). Those who understand: Knowledge growth in teaching. Educational Researcher, 15(2), 4-14. https://doi.org/10.3102/0013189X015002004

Shulman, L. (1987). Knowledge and teaching: Foundations of the new reform. Harvard Educational Review, 57(1), 1-23. https:// doi.org/10.17763/haer.57.1.j463w79r56455411

Smith, A. C., Stewart, R., Shields, P., Hayes-Klosteridis, J., Robinson, P., y Yuan, R. (2005). Introductory biology courses: a framework to support active learning in large enrollment introductory science courses. Cell Biology Education, 4(2), 143-156. https://doi.org/10.1187/cbe.04-08-0048

Thai, N.T.T., De Wever, B., y Valcke, M. (2017). The impact of a flipped classroom design on learning performance in higher education: Looking for the best "blend" of lectures and guiding questions with feedback. Computers E Education, 107, 113-126. https://doi.org/10.1016/j.compedu.2017.01.003

Van den Heuvel-Panhuizen, M., y Drijvers, P. (2020). Realistic mathematics education. Encyclopedia of mathematics education, 713-717. https://doi.org/10.1007/978-3-030-15789-0

Vásquez, C., Alsina, Á., Pincheira, N.G., Gea, M. M., y Chandia, E. (2020). Construcción y validación de un instrumento de observación de clases de probabilidad. Enseñanza de las Ciencias, 38(2), 25-43. https://doi.org/10.5565/rev/ensciencias.2820 


\section{Anexos}

Rúbrica para la valoración de los vídeos

\begin{tabular}{|c|c|c|c|c|}
\hline Componente & Indicador & Baja & Media & Alta \\
\hline \multirow{3}{*}{$\begin{array}{c}\text { Calidad del vídeo } \\
(10 \%)\end{array}$} & Imagen (2 \%) & $\begin{array}{l}\text { Predominan las } \\
\text { imágenes con mala } \\
\text { definición, desenfo- } \\
\text { cadas o pixeladas. } \\
\text { La iluminación im- } \\
\text { pide una correcta } \\
\text { visualización. }\end{array}$ & $\begin{array}{l}\text { La imagen es en ge- } \\
\text { neral nítida y está } \\
\text { bien definida, aun- } \\
\text { que tiene algunos } \\
\text { fallos de ilumina- } \\
\text { ción, pixelados mo- } \\
\text { mentáneos o desen- } \\
\text { foques. }\end{array}$ & $\begin{array}{l}\text { La imagen es nítida, } \\
\text { no hay problemas } \\
\text { apreciables de cali- } \\
\text { dad y definición. }\end{array}$ \\
\hline & Sonido (2 \%) & $\begin{array}{l}\text { Hay tantas interfe- } \\
\text { rencias o interrup- } \\
\text { ciones del sonido } \\
\text { que dificultan enor- } \\
\text { memente la com- } \\
\text { prensión de lo que } \\
\text { se dice. }\end{array}$ & $\begin{array}{l}\text { Hay algunas in- } \\
\text { terferencias o inte- } \\
\text { rrupciones sonoras } \\
\text { que dificultan la es- } \\
\text { cucha, pero mayori- } \\
\text { tariamente es acep- } \\
\text { table. }\end{array}$ & $\begin{array}{l}\text { El sonido es claro, se } \\
\text { escucha sin interfe- } \\
\text { rencias y se entien- } \\
\text { de sin dificultad. }\end{array}$ \\
\hline & Edición (6 \%) & $\begin{array}{l}\text { El vídeo no está bien } \\
\text { editado, las transi- } \\
\text { ciones son bruscas, } \\
\text { con cortes poco cui- } \\
\text { dados. }\end{array}$ & $\begin{array}{l}\text { El vídeo está bien } \\
\text { editado, las transi- } \\
\text { ciones no son brus- } \\
\text { cas, no hay interrup- } \\
\text { ciones en la edición, } \\
\text { pero no tiene una } \\
\text { edición cuidada. }\end{array}$ & $\begin{array}{l}\text { El vídeo está bien } \\
\text { editado, las transi- } \\
\text { ciones no son brus- } \\
\text { cas, no hay interrup- } \\
\text { ciones en la edición, } \\
\text { pero no tiene una } \\
\text { edición cuidada. }\end{array}$ \\
\hline \multirow{3}{*}{$\begin{array}{c}\text { Calidad } \\
\text { del contenido } \\
\text { matemático } \\
(40 \%)\end{array}$} & $\begin{array}{c}\text { Adecuación } \\
\text { del contenido } \\
\text { matemático }(16 \%)\end{array}$ & $\begin{array}{l}\text { Las definiciones y } \\
\text { procedimientos no } \\
\text { están adaptados al } \\
\text { nivel educativo al } \\
\text { que se dirigen. }\end{array}$ & $\begin{array}{l}\text { Las definiciones y } \\
\text { procedimientos se } \\
\text { adaptan parcial- } \\
\text { mente al nivel edu- } \\
\text { cativo al que se di- } \\
\text { rigen, siendo en } \\
\text { algunas ocasiones } \\
\text { de nivel superior o } \\
\text { inferior al nivel se- } \\
\text { leccionado. }\end{array}$ & $\begin{array}{l}\text { Las definiciones y } \\
\text { procedimientos es- } \\
\text { tán adaptados al ni- } \\
\text { vel educativo al que } \\
\text { se dirigen. }\end{array}$ \\
\hline & $\begin{array}{c}\text { Correspondencia } \\
\text { de los estándares } \\
\text { de aprendizaje } \\
(16 \%)\end{array}$ & $\begin{array}{l}\text { El contenido del ví- } \\
\text { deo no se relaciona } \\
\text { con ningún estándar } \\
\text { de aprendizaje eva- } \\
\text { luable y/o estos no } \\
\text { aparecen especifica- } \\
\text { dos en el vídeo. }\end{array}$ & $\begin{array}{l}\text { El contenido mate- } \\
\text { mático del video se } \\
\text { relaciona parcial- } \\
\text { mente con los están- } \\
\text { dares de aprendiza- } \\
\text { je evaluables de los } \\
\text { bloques } 1 \text { y/o } 5 \text { pero } \\
\text { falta especificar de } \\
\text { manera detallada } \\
\text { alguno de los están- } \\
\text { dares. }\end{array}$ & $\begin{array}{l}\text { El contenido mate- } \\
\text { mático del video se } \\
\text { relaciona con los es- } \\
\text { tándares de apren- } \\
\text { dizaje evaluables } \\
\text { de los bloques } 1 \text { y/o } \\
5 \text { especificando de } \\
\text { manera detallada } \\
\text { cada uno de los es- } \\
\text { tándares. }\end{array}$ \\
\hline & $\begin{array}{c}\text { Claridad y preci- } \\
\text { sión matemáticas } \\
\qquad(8 \%)\end{array}$ & $\begin{array}{l}\text { Las matemáticas ex- } \\
\text { plicadas presentan } \\
\text { pérdidas de clari- } \\
\text { dad o de precisión, } \\
\text { o ambigüedades } \\
\text { que dificultan la } \\
\text { comprensión o ge- } \\
\text { neran aprendizajes } \\
\text { erróneos. }\end{array}$ & $\begin{array}{l}\text { Las matemáticas } \\
\text { explicadas tienen } \\
\text { algunos momen- } \\
\text { tos de imprecisión } \\
\text { o ambigüedad, que } \\
\text { no afectan a la com- } \\
\text { prensión global. }\end{array}$ & $\begin{array}{l}\text { Los contenidos ma- } \\
\text { temáticos se desa- } \\
\text { rrollan de manera } \\
\text { clara y precisa y, las } \\
\text { explicaciones están } \\
\text { bien argumentadas. }\end{array}$ \\
\hline
\end{tabular}




\begin{tabular}{|c|c|c|c|c|}
\hline Componente & Indicador & Baja & Media & Alta \\
\hline \multirow{3}{*}{$\begin{array}{l}\text { Calidad } \\
\text { de la transposición } \\
\text { didáctica } \\
(40 \%)\end{array}$} & $\begin{array}{l}\text { Calidad del lenguaje } \\
\text { matemático }(8 \%)\end{array}$ & $\begin{array}{l}\text { El lenguaje matemático } \\
\text { (verbal y escrito) que se } \\
\text { utiliza presenta más de } \\
\text { dos casos de usos inco- } \\
\text { rrectos, inapropiados, } \\
\text { vulgarizados, excesiva- } \\
\text { mente simplificados o } \\
\text { demasiado complejos. }\end{array}$ & $\begin{array}{l}\text { El lenguaje matemático } \\
\text { (verbal y escrito) que se } \\
\text { utiliza es en general cla- } \\
\text { ro, pero hay una o dos } \\
\text { ocasiones en las que se } \\
\text { maneja algún concepto } \\
\text { de manera no rigurosa, } \\
\text { vulgarizado, con un ex- } \\
\text { ceso de simplificación } \\
\text { o complejización o con } \\
\text { una falta de adecuación } \\
\text { al nivel. }\end{array}$ & $\begin{array}{l}\text { El lenguaje matemático } \\
\text { (verbal y escrito) que } \\
\text { se utiliza es claro pero } \\
\text { riguroso, las definicio- } \\
\text { nes y las explicaciones } \\
\text { se manejan adecuada- } \\
\text { mente, adaptándolas al } \\
\text { nivel correspondiente. }\end{array}$ \\
\hline & $\begin{array}{c}\text { Explicación } \\
\text { adecuada al nivel } \\
\text { indicado }(16 \%)\end{array}$ & $\begin{array}{l}\text { La argumentación, el } \\
\text { razonamiento y las ex- } \\
\text { plicaciones presentan } \\
\text { más de un momento de } \\
\text { gran abstracción o com- } \\
\text { plejización, o una sim- } \\
\text { plificación excesiva. } \\
\text { o } \\
\text { No se utilizan contex- } \\
\text { tos y situaciones próxi- } \\
\text { mas al alumnado que le } \\
\text { ayuden a dar sentido a } \\
\text { las matemáticas. }\end{array}$ & $\begin{array}{l}\text { La argumentación, el } \\
\text { razonamiento y las ex- } \\
\text { plicaciones presentan } \\
\text { como mucho una la- } \\
\text { guna en la cual hay un } \\
\text { exceso de abstracción o } \\
\text { un salto conceptual de- } \\
\text { masiado grande o una } \\
\text { simplificación muy ex- } \\
\text { cesiva, aunque se pue- } \\
\text { de considerar que no } \\
\text { afecta al conjunto del } \\
\text { vídeo. Se utilizan con- } \\
\text { textos y situaciones } \\
\text { próximas. }\end{array}$ & $\begin{array}{l}\text { La argumentación, el } \\
\text { razonamiento y las ex- } \\
\text { plicaciones que se pro- } \\
\text { porcionan parten del } \\
\text { conocimiento previo } \\
\text { del alumnado ayudán- } \\
\text { dole a construir el nue- } \\
\text { vo, utilizando contextos } \\
\text { próximos y situaciones } \\
\text { y explicaciones asequi- } \\
\text { bles, sin caer en una ex- } \\
\text { cesiva simplificación. }\end{array}$ \\
\hline & $\begin{array}{l}\text { Recursos utilizados } \\
\qquad(16 \%)\end{array}$ & $\begin{array}{l}\text { No se utiliza ningún } \\
\text { recurso manipulativo } \\
\text { ni tecnológico. } \\
\text { o } \\
\text { Se utiliza algún recur- } \\
\text { so, pero no es el apro- } \\
\text { piado para la explica- } \\
\text { ción o el problema que } \\
\text { se aborda. }\end{array}$ & $\begin{array}{l}\text { Se utiliza algún recurso } \\
\text { manipulativo o tecno- } \\
\text { lógico, pero no queda } \\
\text { clara su finalidad en el } \\
\text { contexto del problema } \\
\text { o el contenido matemá- } \\
\text { tico que se está tratan- } \\
\text { do. Se aprecia inten- } \\
\text { ción didáctica, pero no } \\
\text { se aprovecha suficiente- } \\
\text { mente. }\end{array}$ & $\begin{array}{l}\text { Se utilizan recursos ma- } \\
\text { nipulativos (físicos o } \\
\text { virtuales), tecnológicos } \\
\text { o de otra índole para } \\
\text { ayudar a construir los } \\
\text { conceptos matemáti- } \\
\text { cos que se trabajan, re- } \\
\text { presentar el problema o } \\
\text { buscar la solución. Son } \\
\text { adecuados para la eta- } \\
\text { pa correspondiente. }\end{array}$ \\
\hline \multirow[t]{2}{*}{$\begin{array}{l}\text { Ampliaciones } \\
\qquad(10 \%)\end{array}$} & $\begin{array}{l}\text { Nivel de interacción } \\
\qquad(4 \%)\end{array}$ & $\begin{array}{l}\text { No se realiza interac- } \\
\text { ción. }\end{array}$ & $\begin{array}{l}\text { Se plantean activida- } \\
\text { des y preguntas, pero } \\
\text { no se comprueban las } \\
\text { respuestas. }\end{array}$ & $\begin{array}{l}\text { Requiere que el alum- } \\
\text { nado tenga respuesta } \\
\text { activa: realizar tareas, } \\
\text { responder preguntas, } \\
\text { etc. Se comprueba la } \\
\text { respuesta (test, posi- } \\
\text { bles respuestas, "si has } \\
\text { hecho esto" ...). }\end{array}$ \\
\hline & $\begin{array}{c}\text { Calidad } \\
\text { de la interacción }(6 \%)\end{array}$ & $\begin{array}{l}\text { No se realiza interac- } \\
\text { ción. }\end{array}$ & $\begin{array}{l}\text { La interacción se realiza } \\
\text { planteando verbalmen- } \\
\text { te preguntas. }\end{array}$ & $\begin{array}{l}\text { La interacción se reali- } \\
\text { za utilizando software } \\
\text { específico. }\end{array}$ \\
\hline
\end{tabular}

